\title{
SHORT AND LONG TERM FOLLOW UP RESULTS OF (THE VERSATILE) GASTROCNEMIUS MUSCLE FLAP
}

\author{
Ghazanfar Ali, Shahid Hameed*, Abdul Majid**, Rao Saud Ahmad*, Ahsan Masood Butt ${ }^{* * *}$, Rana Hassan Javed****
}

Combined Military Hospital Quetta/National University of Medical Sciences (NUMS) Pakistan, *Combined Military Hospital/National University of Medical Sciences (NUMS) Rawalpindi Pakistan, ${ }^{* *}$ Combined Military Hospital Pano Aqil/National University of Medical Sciences (NUMS) Pakistan, ${ }^{* * *}$ Combined Military Hospital Lahore/National University of Medical Sciences (NUMS) Pakistan, ${ }^{* * * *}$ Combined Military Hospital Peshawar/National University of Medical Sciences (NUMS) Pakistan

\begin{abstract}
Objective: To see the long and short term outcome of the Gastrocnemius muscle flap for coverage of soft tissue defects of knee and proximal Tibia.

Study Design: Case series.

Place and Duration of Study: Surgery Department of Combined Military Hospital Bahawalpur, from Dec 2014 to Oct 2017.

Methodology: A total of 33 patients were enrolled for the study through consecutive sampling. Data was processed using SPSS-17.

All patients were followed up at first and second week (early morbidity), and then at 06 months (late morbidity) from operation. During early follow up, they were examined for Hematoma at donor site, wound Infection, sural nerve injury, partial skin graft loss and Partial Flap Necrosis. In late follow up patients were examined for reduced Knee mobility and Gastrocnemius muscle contractions.

Results: The studied 33 patients consisted of 30 (90.90\%) men and $3(9.09 \%)$ women. The mean age was 35 years (range: 16 to 64) years. Hematoma was commonest complication. It occurred in $6.06 \%$. Wound infection, partial loss of flap, partial loss of skin graft and wound infection occurred in $3.03 \%$ patients.

Late follow up revealed reduced knee mobility in $9.09 \%$ patients $(n=3)$ and bothersome contraction of gastrocnemius muscle in same number of patients.

Conclusion: The gastrocnemius flap is reliable, versatile, and very useful for provision of supple soft tissue coverage for defects around the knee and the upper leg.
\end{abstract}

Keywords: Gastrocnemius Muscle, Knee joint, Type 1 Flaps.

This is an Open Access article distributed under the terms of the Creative Commons Attribution License (http://creativecommons.org/licenses/by/4.0), which permits unrestricted use, distribution, and reproduction in any medium, provided the original work is properly cited.

\section{INTRODUCTION}

Lower Limb trauma constitutes a major chunk of patients in almost all medical setups dealing with surgical patients ${ }^{1}$. It can be attributed to increased number of road traffic accidents due to over speeding and violation of traffic rules. Plastic surgeons are often consulted to provide soft tissue coverage in lower limb trauma patients having exposed bone, joint or main vessels.

There are number of options for provision of soft tissue coverage in defects around knee joint ${ }^{2}$. Free tissue transfer requires team approach and long operative time, donor site morbidity, and a definitive risk of total failure and prolonged

Correspondence: Dr Ghazanfar Ali, Plastic Surgeon, Combined Military Hospital, Quetta Pakistan

Received: 23 Nov 2018; revised received: 04 Dec 2020; accepted: 24 Dec 2020 hospital stay as compared to local rotation flaps ${ }^{3}$. Gastrocnemius Muscle rotation flap is one option amongst local flaps for soft tissue coverage. Gastrocnemius Muscle is a Type I flap and has a robust blood supply ${ }^{4}$. The dominant vascular pedicle is sural artery, a muscular Branch of Popliteal Artery. Due to its constant anatomy and ease of harvest it is a workhorse flap for coverage of soft tissue defects around the $\mathrm{knee}^{5}$. It can be used as myocutaneous flap or Muscle only flap along with split thickness skin graft (STSG). It can cover areas of distal femor or proximal tibia. However, additional mobility of the muscle can be achieved by mobilizing its origin from distal femor.

\section{Vascular Anatomy}

This is classified as Mathe's and Nahai Type I Muscle Flap. Type I muscle flaps are supplied 
by a single artery ${ }^{4}$. The vascular pedicle comprises of sural artery (formerly gastrocnemius artery) and of one or two veins. In a certain number of cases, a secondary sural artery is present ${ }^{6}$. The vascular pedicle is well protected within the popliteal fossa. The average length of sural artery is $4 \mathrm{~cm}$. The diameter of the sural arteries is 2 to 2.5 $\mathrm{mm}$ on average ${ }^{7}$. In most cases, the sural artery terminates into 2 branches, occasionally 3 or even 4, having a longitudinal course, in the muscle axis, just below its deep aspect and a terminal vascular distribution ${ }^{8}$. These arteries give rise to the cutaneous perforating branches. There is anastomosis with opposite Gastrocnemius and soleus Muscle. These vascular arrangements allow surgeon to raise this flap as myocutaneous flap or distally based flap.

\section{Surgical Technique}

Pre-operatively the Patients were counseled about the procedure in detail. They were informed about the nature of operation, duration of hospitalization, possible adverse outcomes; follow up in outpatient department (OPD). These procedures were carried out in General or Spinal anesthesia depending upon patient conditions. Tourniquet was used in all cases and pressure was kept $100 \mathrm{~mm}$ of $\mathrm{Hg}$ above systolic blood pressure.

After general or spinal anesthesia surgical site was prepared with povidone solution and recipient site was prepared by debridement and defect size measured. For medial half of muscle incision was made on medial side while lateral half was approached through posterior incision. Gastrocnemius muscle was identified and separated from soleus with the help of plantaris tendon.

After careful dissection and hemostasis the medial or lateral half of the gastrocnemius muscle was placed over the defect and stabilized with vicryl sutures followed by STSG. Drain was placed at donor site to avoid hematoma formation. First dressing was changed after 4-5 days.

\section{METHODOLOGY}

It was a cohort study carried out at the Plastic and Reconstructive Surgery Department of Combined Military Hospital Bahawalpur, from December 2014 to October 2017 after approval from the hospital research and ethical committee. In total 33 patients reporting to the department for provision of soft tissue coverage to knee or proximal leg wounds were enrolled for the study through consecutive sampling. Patients with Knee arthritis, diabetes mellitus, and a history of smoking were excluded.

Before enrolling the patients were counseled about the procedure in detail and possible adverse outcomes. History of present illness, previous treatment, occupation, pre-existing pain, diabetes mellitus and smoking was obtained and recorded in especially designed proformas. Data was processed using SPSS-17. During patient selection, three patients were excluded from the study. One patient had diabetic neuropathy, and two patients had severe knee arthritis.

All patients were followed up at first and second week (early morbidity), and then at 06 months (late morbidity) from operation. During early follow up, they were examined for Hematoma at donor site, wound Infection, sural nerve injury, partial skin graft loss and Partial Flap Necrosis. In late follow up patients were examined for reduced Knee mobility and Gastrocnemius muscle contractions.

\section{RESULTS}

The studied 33 patients consisted of 30 $(90.90 \%)$ men and $3(9.10 \%)$ women. The mean age was 35 years (range: 16 to 64) years. In early follow up hematoma formation was seen at donor site in $6.06 \%$ patients $(n=2)$. This was followed by surgical site infection, partial loss of skin graft

Table-I: Early follow up.

\begin{tabular}{|c|c|}
\hline Complications & n ( $\%)$ \\
\hline Hematoma at donor site & $2(6.06 \%)$ \\
\hline Wound Infection & $1(3.03 \%)$ \\
\hline Sural nerve injury & - \\
\hline Partial Skin graft loss & $1(3.03 \%)$ \\
\hline Partial flap necrosis & $1(3.03 \%)$ \\
\hline \multicolumn{2}{|l|}{ Table-II: Late follow up. } \\
\hline Complications & n (\%) \\
\hline Reduced Knee Mobility & $3(9.09 \%)$ \\
\hline Gastrocnemius Muscle Contractions & $3(9.09 \%)$ \\
\hline
\end{tabular}


and partial loss of flap. All these complications occurred in $3.03 \%$ patients $(n=1)$. Sural nerve injury did not occur in any patient (table-I).

Late follow up revealed reduced knee mobility in $9.09 \%$ patients $(n=3)$ and bothersome contraction of gastrocnemius muscle in same number of patients (table-II).
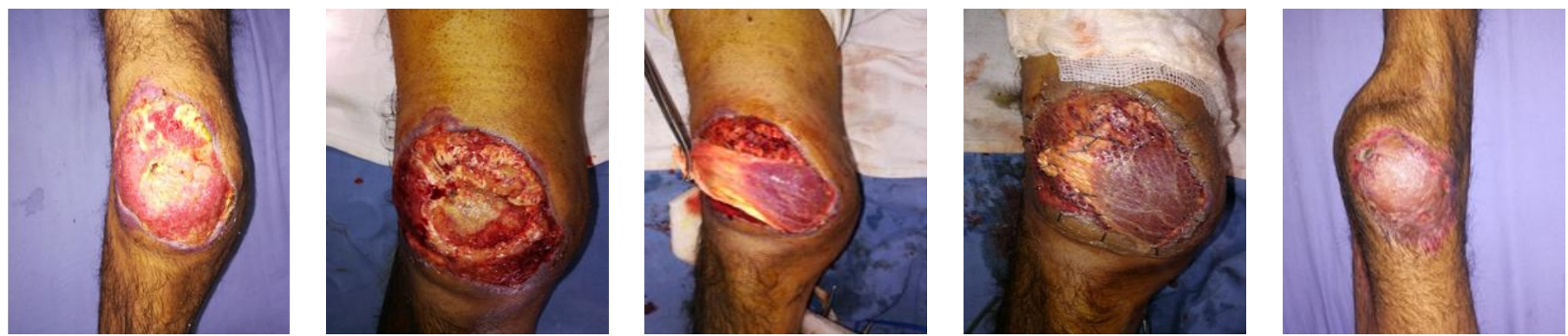

The management of high-energy lower extremity trauma with soft tissue and bone injuries remains a challenge. These injuries often occur in the poly trauma patient making management even more difficult. The tools in the armamentarium of plastic surgeon include skin grafting, local flaps, perforator flaps, muscle flaps, micro-

\section{DISCUSSION}

The lower extremities are mechanically designed by the nature to bear weight. Moreover, with advancing age there are attritional changes which aggravate make reconstruction more difficult. These functional requirements make an effective reconstruction difficult. The lower extremity defects results from trauma, diabetes and vascular disease, cancer ablation, and other disease processes. The importance of thorough understanding of all plastic surgical tools and good surgical hand cannot be over emphasized.

The main objective in the treatment of open tibial fractures and lower extremity salvage is to preserve a limb that will be more functional than if it was amputated 9 . If the extremity cannot be salvaged, the goal is to maintain the maximum functional length. This is particularly important with respect to the knee joint. If the knee unit is salvageable, a below-knee amputation is performed as the work of ambulation is significantly reduced in patients with below-knee amputations when compared with patients with above-knee amputations. Patients with below knee amputations have a more normal gait and a greater ability to perform more physical activities than patients with above-knee amputations ${ }^{10,11}$. vascular free flaps, neurovascular repair and bone stabilization. The decision to choose the best options out of many available depends upon the multiple factors like; etiology of defect, size and shape of defect, clinical condition of the patient, presence of co-morbidities, available health facilities and experience of the surgeon ${ }^{12}$.

Gastrocnemius flap was used initially for coverage of osteomyelitis and similar problems ${ }^{13}$. Over the time various modifications in composition of flap were made as per requirements ${ }^{14,15}$.

Although the Gastrocnemius muscle flap is workhorse flap in provision of soft tissue coverage for soft tissue defects of knee and proximal tibia, yet there are some adverse outcomes in this process. In this study early follow up revealed formation of hematoma at donor site in 2 (6.06\%) patients. This happened in spite of using suction drain. In both cases it settled without surgical intervention. Although it is not a very high figure, it should be avoided in all cases. Meticulous surgical technique and proper hemostasis can help avoid this problem. Only $1(3.03 \%)$ patients had surgical site infection, partial loss of flap and partial loss of skin graft. Fortunately there was no case of sural nerve injury. Contemporary studies show similar outcome of this flap. 
Hendy et al conducted a study in Egypt revealed partial necrosis of the flap in two cases $(20 \%)$, formation of hematoma in one case $(10 \%)$ and surgical site infection in two cases $(20 \%)^{16}$.

A recent study by Abd-Almoktader et al revealed similar results. There was one case with postoperative hematoma and one case with wound dehiscence healed by local wound care, one case with lost skin graft needed re-grafting17.

Long term follow up in our study revealed reduced knee movement and gastrocnemius muscle contractions in $3(9.09 \%)$ patients. The former improved with physiotherapy and excercises.

The gastrocnemius muscle flap is probably one of the safest flaps, yet it can result in some functional problems. Daigeler et al, reported one complete flap loss in only one case of 218 patients $(0.46 \%)$ and $87 \%$ of patients were not significantly crippled for walking on even ground, but only $42 \%$ of patients could run and $40 \%$ of patients complained about pain when walking more than 200 meters $^{18}$.

\section{CONCLUSION}

The gastrocnemius flap is reliable, versatile, and very useful for provision of supple soft tissue coverage for defects around the knee and the upper leg.

\section{CONFLICT OF INTEREST}

This study has no conflict of interest to declare by any author.

\section{REFERENCES}

1. Ali G, Hameed S, Javed RH, Yousaf O, Almas D. Short and long term follow up results of (The Versatile) reverse sural artery flap. Pak Armed Forces Med J 2015; 65(1): 58-62.
2. Levin LS. The reconstructive ladder. An orthoplastic approach. Orthop Clin North Am 1993; 24(3): 393-409.

3. Le Nen D, Stin Del E, Carol P. Therapeutic possibilities of the lateral supramalleolar flap and itsvariations: Apropos of 6 clinical cases. Ann Chir Plast Esthet 1996; 41(2): 127-35.

4. Mathes SJ, Nahai F. Classification of the vascular anatomy of muscles: experimental and clinical correlation. Plast Reconstr Surg 1981; 67(2): 177-87.

5. Breidenbach WC, Terzis, JK. The anatomy of free vascularized nerve grafts. Clin Plast Surg 1984; 11(1): 65-71.

6. Mairesse JL, Mestdagh H, Procyk S. Contribution a 'l'e 'tude de la vascularisation arte 'rielle du muscle triceps sural. Anat Anz 1984; 155(1-5): 195-202.

7. Tropet Y, Nicolet F, Guyot J. Le lambeau libre du jumeau interne. Ann Chir Plast Esthet 1985; 30(4): 316-20.

8. Smrcka V, Stingl J, Kubin K. Anatomical notes on gastrocnemius muscle uses for muscle flap preparation. Acta Chir Plast 1986; 28(2): 112-20.

9. Rodriguez ED, Bluebond-Langner R, Copeland C, et al. Functional outcomes of posttraumatic lower limb salvage: a pilot study of anterolateral thigh perforator flaps versus muscle flaps. J Trauma 2009; 66(5): 1311-14.

10. Burger H, Marincek C. Return to work after lower limb amputation. Disabil Rehabil 2007; 29(17): 1323-29.

11. Bosse MJ, MaCKennzie EJ, Kellam JF, Burgess AR, Webb LX, Swiontkowski MF, et al. An analysis of outcomes of reconstruction or amputation after leg-threatening injuries. N Eng J Med 2002; 347(24): 1924-31.

12. Anthony JP, Mathes SJ, Alpert BS. The muscle flap in the treatment of chronic lower extremity osteomyelitis: results in patients over 5 years after treatment. Plast Reconstr Surg 1991; 88(2): 311-18.

13. Ger R. The operative treatment of the advanced stasis ulcer: a preliminary communication. Am J Surg 1966; 11: 659-63.

14. Orticochea M. The musculo-cutaneous flap method: an immediate and heroic substitute for the method of delay. Br J Plast Surg 1972; 25: 106.

15. Mac-Craw JB, Fishman JH, Sharzer LA (1978) The versatile gastrocnemius myocutaneous flap. Plast Reconst Surg 1978; 62(1): 15-23.

16. Hendy A, Allam A, Abdel-Khalek A, Zayed E, Abdel-Razek S, El-Melegy N, et al. Split Gastrocnemius Muscle Flap; Egypt. J Plast Reconstr Surg 2003; 27(2): 181-87.

17. Abd-Almoktader MA. Gastrocnemius muscle flap for middle third leg defects. Egypt J Plast Reconstr Surg 2014; 38(2): 197204.

18. Daigeler A, Drücke D, Tatar K, Homann HH, Goertz O, Tilkorn $\mathrm{D}$, et al. The pedicled gastrocnemius muscle flap: a review of 218 cases. Plast Reconstr Surg 2009; 123(1): 250-57. 